\title{
Role of Coherent Structures in Turbulent Premixed Flame Acoustics
}

\author{
D. Brouzet *, A. Haghiri ${ }^{\dagger}$, M. Talei ${ }^{\ddagger}$, M. J. Brear ${ }^{\S}$ \\ University of Melbourne, Parkville VIC, 3010, Australia \\ O. T. Schmidt ${ }^{\text {II }}$ \\ University of California San Diego, La Jolla, CA 92093 \\ G. Rigas" , T. Colonius** \\ California Institute of Technology, Pasadena, CA 91125
}

https://doi.org/10.2514/1.J058480

Spectral proper orthogonal decomposition (SPOD) is applied to direct numerical simulation (DNS) datasets of a lean and a stoichiometric methane/air turbulent premixed jet flame. SPOD is used to extract the coherent structures that correlate with the radiated sound by using an inner product based on a linearized disturbance energy. Two types of structures are prominent in the data. The first type arises in the jet's shear layer and is linked to the Kelvin-Helmholtz (K-H) instability, which is an important mechanism of sound generation in non-reacting jets. These structures produce sound through deformation of the flame front in the shear layer. They contain most of the acoustic energy and are dominant at Strouhal numbers (defined based on the jet's diameter and the inlet mean velocity) less than unity. The second type of structures is found near the jet centreline, where large fluctuations of the flame surface are observed. The structures are linked to small non-linear flame dynamics and to the Orr mechanism. They travel at a speed close to the inlet mean velocity and are important at higher Strouhal numbers. Regardless of their energy content, both types of structures have important contributions to the broadband nature of combustion noise.

\section{Nomenclature}

$c \quad=\quad$ speed of sound, $\mathrm{m} / \mathrm{s}$

\footnotetext{
*PhD Student, Department of Mechanical Engineering.

${ }^{\dagger}$ Postdoctoral Researcher, Department of Mechanical Engineering.

¥Senior Lecturer, Department of Mechanical Engineering.

${ }^{\S}$ Professor, Department of Mechanical Engineering.

IIAssistant Professor, Mechanical and Aerospace Engineering, Member AIAA.

" Postdoctoral Research Associate, Division of Engineering and Applied Science.

**Professor, Division of Engineering and Applied Science, Associate Fellow AIAA.

Copyright $\odot 2020$ by the American Institute of Aeronautics and Astronautics, Inc. All rights reserved. All requests for copying and permission to reprint should be submitted to CCC at www.copyright.com; employ the eISSN 1533-385X to initiate your request. See also AIAA Rights and Permissions www.aiaa.org/randp.
} 


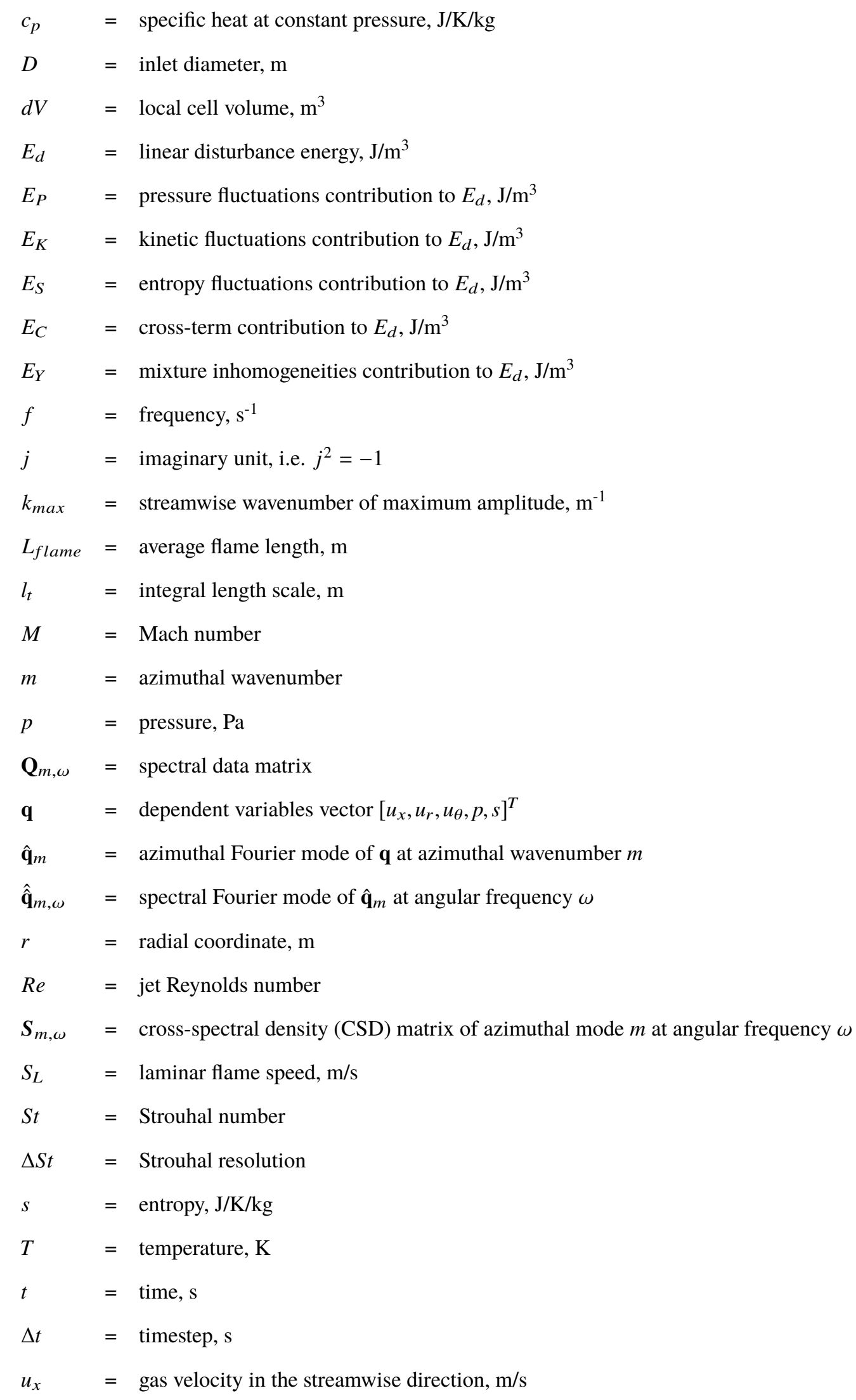




$$
\begin{aligned}
& u_{r} \quad=\text { gas velocity in the radial direction, } \mathrm{m} / \mathrm{s} \\
& u_{\theta}=\text { gas velocity in the azimuthal direction, } \mathrm{m} / \mathrm{s} \\
& \mathbf{u}=\text { vector of gas velocities in the streamwise, radial and azimuthal directions } \\
& v_{p} \quad=\text { phase velocity, } \mathrm{m} / \mathrm{s} \\
& \mathbf{W}=\text { weighting matrix } \\
& x=\text { streamwise coordinate, } \mathrm{m} \\
& \mathbf{x}=\text { vector of streamwise, radial and azimuthal coordinates } \\
& \gamma \quad=\quad \text { specific heat capacity ratio } \\
& \delta_{t h}=\text { thermal flame thickness, } \mathrm{m} \\
& \lambda^{(i)}=\text { eigenvalue of } i^{t h} \text { mode } \\
& \boldsymbol{\Lambda}_{m, \omega}=\text { diagonal eigenvalues matrix of azimuthal mode } m \text { at angular frequency } \omega \\
& \theta \quad=\text { azimuthal coordinate, } \mathrm{rad} \\
& v \quad=\text { gas kinematic viscosity, } \mathrm{m}^{2} / \mathrm{s} \\
& \rho \quad=\text { gas density, } \mathrm{kg} / \mathrm{m}^{3} \\
& \boldsymbol{\Phi}_{m, \omega}^{(i)}=i^{t h} \text { SPOD mode of azimuthal mode } m \text { at angular frequency } \omega \\
& \phi \quad=\text { equivalence ratio } \\
& \boldsymbol{\Psi}_{m, \omega}=\text { orthogonal eigenvectors matrix of azimuthal mode } m \text { at angular frequency } \omega \\
& \Omega \quad=\text { acoustic sub-domain in the SPOD formulation, } \mathrm{m}^{3} \\
& \omega \quad=\text { angular frequency }
\end{aligned}
$$

Subscripts

ad = adiabatic quantities

$q_{b} \quad=\quad$ baseflow solution of $q$

$q_{f} \quad=\quad$ first order fluctuations of $q$

ref $=$ inlet quantities

\section{Modifiers}

$$
\begin{array}{ll}
\mathbf{q}^{(n)} & =n^{t h} \text { realization of vector } \mathbf{q} \\
\mathbf{q}^{T} & =\text { transpose of vector } \mathbf{q} \\
\mathbf{q}^{*} & =\text { Hermitian transpose of vector } \mathbf{q} \\
\bar{q} & =\text { temporal average of } q \\
q^{\prime} & =\text { temporal fluctuations of } q \\
\|q\| & =\text { time averaged volume norm of } q
\end{array}
$$




\section{Introduction}

I NDUSTRIAL gas turbines are an important form of power generation. It is well known that nitrogen oxide emissions from these devices can be reduced by operating at lean premixed conditions. However, this can make the combustor susceptible to thermo-acoustic instabilities, which are commonly initiated by combustion noise and can lead to combustor failure in extreme cases [1, 2]. Improving our understanding of sound generated by turbulent flames is therefore an important step towards developing safer and low emission gas turbines.

Several experimental studies of open, turbulent, premixed and diffusion flames have demonstrated the broadband nature of combustion noise [3-6] for a large variety of geometries and flow variables. A recent study by Tam [7] compared experimental hydrocarbon combustion noise spectra from turbulent open flames, low Mach number jets, can-type combustors, auxiliary power units and turbofan engines. This study verified that combustion noise broadband spectrum can be satisfactory modeled by the similarity spectrum of the noise from large turbulent structures in high speed jets. This is consistent with an earlier explanation provided by Strahle [8], who argued that the frequency content of combustion noise is governed by large scale motions of the turbulence, through distortions of the flame surface.

Spatio-temporal deformations of the flame surface are characterized by fluctuations of the overall heat release rate, which have been demonstrated to be the dominant source of sound generation in open, low Mach number, turbulent premixed flames, e.g. [2, 9]. Moreover, particular flame and flow structures have been associated with fluctuations of the heat release rate. For instance, vortices in laminar premixed V-flames [10] and turbulent premixed slot jet flames [11] were found to strongly wrinkle the flame front and produce sound. Flame annihilation events such as flame pinch-off and consumption of fresh gas pockets were also shown to be responsible for sound generation in experimental [10, 12] and numerical [13,-16] studies of premixed flames. All these previous studies considered the heat release rate fluctuations as the primary source of noise. However, this approach does not provide a direct link between all flow or flame features in the near field and the produced sound in the far-field.

Modal decomposition techniques such as proper orthogonal decomposition (POD) or dynamic mode decomposition (DMD) have been extensively used to identify coherent structures in turbulent flows [17]. Typically, POD is used to decompose the data into spatially orthogonal, time-independent modes, whereas DMD associates a particular frequency and growth rate to each mode and can be seen as a temporal orthogonalization of the data. Both of these techniques have been used in reacting flows, providing insights into coherent structures which contribute to the sound generation mechanism. For instance, Kabiraj et al. [18] suggested that the broadband nature of combustion noise could be due to the high level of unsteadiness in the coherent structures. Other works, such as those by Ghani et al. [19] and Aoki et al. [20], used DMD to identify the dominant modes which are linked to combustion instabilities.

One shortcoming of the classical POD is that the identified modes do not necessarily evolve coherently in time. While DMD adresses this drawback, the modes obtained will be different for different flow realizations. Spectral proper orthogonal decomposition (SPOD) addresses these issues by finding the spatially orthogonal modes oscillating at a 
single frequency. These modes are obtained by solving an eigenvalue problem in spectral space, where the inner product is based on a set of realizations of the temporal Fourier transform. It has been argued that SPOD modes are optimally averaged DMD modes [21].

SPOD was found to be effective for extracting wavepacket structures in non-reacting turbulent jets [22, 23]. Wavepackets are specific spatio-temporal structures which their size exceeds the integral length of the flow. Their generic shape can be described as alternating regions of positive and negative fluctuations, coupled with a modulating spatial envelop that changes their local amplitude. As explained by Jordan \& Colonius [24], their subsonic advection will lead to a small energy leakage to the acoustic far-field and they have been shown to explain the low-frequency $\left(S t=f D / \bar{u}_{r e f}<1\right)$ sound radiation of turbulent non-reacting jets. The wavepacket structures arising from the shear layer of turbulent jets have also been linked to the Kelvin-Helmholtz (K-H) instability [25]. Recently, studies have used SPOD to analyze these structures in non-reacting high-speed jets and their contribution to the far-field sound [26, 27]. For instance, through eigenvalue analysis, Schmidt et al. [27] determined that the axisymmetric SPOD modes had a low-rank behavior (i.e. the dominant coherent structures contained a considerable portion of the total energy) in the frequency band $0.2<S t<2$, peaking at $S t=0.6$ for a subsonic jet.

More works have recently been investigating the importance of non-linearities in turbulent jets. Using SPOD and resolvent analysis, Schmidt et al. [27] have identified another type of wavepacket structure, which was prominent downstream of the potential core. Their interpretation is notably built on the work of Tissot et al. [28, 29], calling these structures Orr-type wavepackets. Tissot et al. [28, 29] suggested that the Orr-type wavepackets were the result of non-modal growth, seeded by non-linearities through the Orr mechanism. However, these structures have not been linked to sound generation, contrary to the K-H wavepackets.

Overall, SPOD is an advanced modal method which has been used to study energetically dominant structures in non-reacting turbulent flows. However, to the best of the authors' knowledge, this technique has not been used in the context of reacting flows acoustics yet. In this work, a suitable SPOD formulation to study reacting flows acoustics is first defined. This is achieved by defining an inner product based on a 'disturbance energy' [30]. Then, SPOD is applied to two direct numerical simulation (DNS) datasets of open turbulent premixed flames generating sound. The chosen datasets were obtained in another study [15] and feature two turbulent jet flames with different equivalence ratios $\phi$ of 0.7 and 1 and therefore different radiated sound amplitudes. SPOD is used to identify the flame and hydrodynamic structures responsible for sound generation in a wide frequency range, and therefore contributing to the broadband nature of combustion noise.

\section{DNS dataset}

DNS datasets of two turbulent premixed flames featuring sound generation are used in this study [15]. The DNSs were performed with a modified version of S3D [31] using simple chemistry, referred to as S3D-SC [32]. A single 


\begin{tabular}{lcc}
\hline \hline & $\phi=1$ & $\phi=0.7$ \\
\hline Inlet jet diameter (D) & $3.94 \mathrm{~mm}$ & $4.63 \mathrm{~mm}$ \\
Domain size & $25 \mathrm{D} \times 16 \mathrm{D} \times 16 \mathrm{D}$ \\
Mean inlet Mach number $\left(M=\bar{u}_{r e f} / c_{r e f}\right)$ & 0.35 & 0.26 \\
Jet Reynolds Number $(R e)$ & 5300 & 5300 \\
Inlet turbulent intensity $\left(u^{\prime} / \bar{u}_{r e f}\right)$ & $3.7 \%$ & $3.7 \%$ \\
Ambient temperature $\left(T_{a d}\right)$ & $2464 \mathrm{~K}$ & $2208 \mathrm{~K}$ \\
Thermal flame thickness $\left(\delta_{t h} / D\right)$ & 0.07 & 0.07 \\
Flame length $\left(L_{\text {flame }} / D\right)$ & 13 & 12 \\
Ratio $u^{\prime} / S_{L}$ & 3.08 & 2.94 \\
Ratio $l_{t} / \delta_{t h}$ & 4.28 & 4.28 \\
Streamwise grid resolution & $8 \mathrm{pts} / \delta_{t h}$ & $8 \mathrm{pts} / \delta_{t h}$ \\
Transverse grid resolution & $14 \mathrm{pts} / \delta_{t h}$ & $14 \mathrm{pts} / \delta_{t h}$ \\
\hline \hline
\end{tabular}

Table 1 Main DNS parameters.

step, irreversible chemistry model based on Arrhenius' law was used to reduce the computational cost. The single-step model used matched several important parameters including the laminar flame speed, the flame thickness and the adiabatic temperature. The set-up consists of a round jet of unburned premixed mixture (reactant) at $T_{\text {ref }}=800 \mathrm{~K}$ issuing into an open environment of combustion products at the adiabatic flame temperature. Two flames are analyzed with equivalence ratios $\phi$ of 1 and 0.7. The jet Reynolds number $R e=\bar{u}_{r e f} D / v_{r e f}$ is equal to 5300 and the inlet Mach number $M=\bar{u}_{r e f} / c_{r e f}$ is equal to 0.35 and 0.26 for the $\phi=1$ and $\phi=0.7$ cases, respectively. A modified synthetic turbulence field, with a turbulence intensity of $3.7 \%$ at the jet centreline, was fed into the mean velocity field using the Taylor hypothesis. Considering the values of $u^{\prime} / S_{L}$ and $l_{t} / \delta_{t h}$ shown in table 1 , we note that the flames lie in the 'thin reaction zone' in the modified combustion regime diagram proposed by Peters [33].

The simulations were performed on a three-dimensional (3-D) structured Cartesian mesh. The grid was uniform inside the jet region including the flame, i.e. for $|y, z|<1.5 D$. For $|y, z|>1.5 D$, a stretched mesh with an increasing grid spacing $\left[\left(\Delta_{i+1} / \Delta_{i}\right)-1\right]$ lower than $2 \%$ was used. Extensive mesh-independence studies were performed to ensure that the flame was adequately resolved and the radiated sound was free of numerical noise.

Table 1 summarizes the main DNS parameters and Fig. 1 shows the computational domain, as well as the iso-surface of the maximum reaction rate at an instant for the $\phi=1$ flame. More details about the computational approach and the chemistry model can be found in Haghiri et al. [15]. Approximately 1.3 jet flow-through times are used for post-processing. The turbulent datasets consist of 700 and 1000 flow-field snapshots for the $\phi=1$ and $\phi=0.7$ cases respectively, with a dimensionless time step of $\Delta t c_{r e f} / D=0.1$. The data is interpolated onto a $1205 \times 519 \times 512$ radially non-uniform cylindrical grid such that $\mathbf{x}=[x, r, \theta] \in[0,20 D] \times[0,8 D] \times[0,2 \pi]$, where $[x, r, \theta]$ represent the streamwise, radial and azimuthal directions, respectively. 


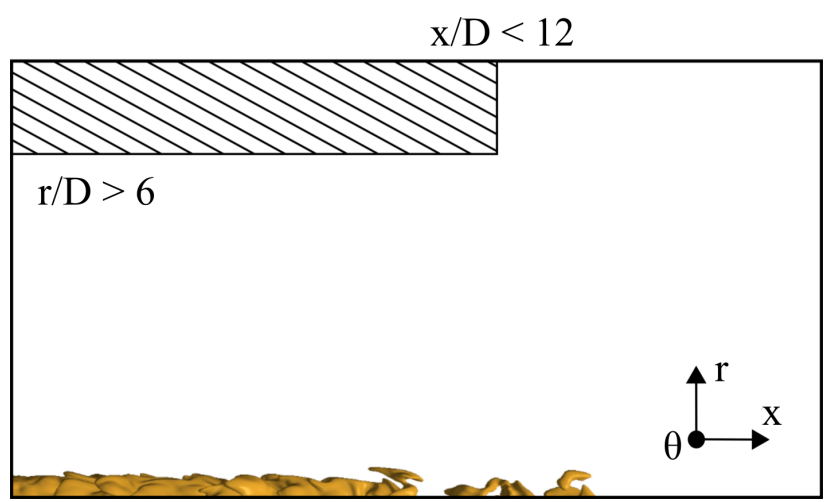

Fig. 1 Iso-surface of maximum reaction rate at an instant for the $\phi=1$ turbulent premixed flame. The hashed region represents the acoustic sub-domain $\Omega$ considered in the SPOD analysis.

\section{Post-processing methods}

\section{A. SPOD formulation}

To perform the SPOD, the dependent variables vector $\mathbf{q}(\mathbf{x}, t)=\left[u_{x}, u_{r}, u_{\theta}, p, s\right]^{T}(\mathbf{x}, t)$ is first decomposed as a sum of a time mean $\overline{\mathbf{q}}(\mathbf{x})$ and a fluctuating part $\mathbf{q}^{\prime}(\mathbf{x}, t)$. The fluctuating component is then decomposed into Fourier modes $\hat{\mathbf{q}}_{m}$ in the azimuthal direction so that,

$$
\mathbf{q}^{\prime}(x, r, \theta, t)=\sum_{m} \hat{\mathbf{q}}_{m}(x, r, t) e^{j m \theta},
$$

where $m$ is the azimuthal wavenumber. This decomposition enables different azimuthal modes $\hat{\mathbf{q}}_{m}$ to be analyzed separately. A further Fourier decomposition in the time domain is performed to obtain $\hat{\mathbf{q}}_{m, \omega}$ at the angular frequency $\omega=2 \pi S t$, where the Strouhal number $S t=f D / \bar{u}_{r e f}$ :

$$
\hat{\mathbf{q}}_{m}(x, r, t)=\sum_{\omega} \hat{\mathbf{q}}_{m, \omega}(x, r) e^{j \omega t}
$$

The temporal dataset is divided into 8 realizations, producing subsets of 256 and 384 timesteps for the $\phi=1$ and $\phi=0.7$ cases, respectively. A Hanning window with $75 \%$ overlap is used for computation of the spectra. The realizations in the SPOD formulation are analogous to the overlaping windows used in Welch's method to compute the power spectral density of a signal [34]. The obtained spectra for each realization have a frequency resolution of $\Delta S t \approx 0.1$ for both cases. Segmenting the data into more realizations leads to better convergence of the SPOD modes, at the cost of decreasing the frequency resolution. For the frequencies presented in this paper, the spatial shape, amplitude and eigenvalues of the two dominant SPOD modes found with 8 and 29 realizations were nonetheless similar. Use of a different windowing function or a different overlap value was not found to affect significantly the results either.

SPOD is then applied to extract the coherent structures of the spectral data matrix $\mathbf{Q}_{m, \omega}=\left[\hat{\mathbf{q}}_{m, \omega}^{(1)}, \hat{\mathbf{q}}_{m, \omega}^{(2)}, \ldots, \hat{\mathbf{q}}_{m, \omega}^{(n)}\right]$ 
, where the superscript ${ }^{(n)}$ represents the $n^{\text {th }}$ realization. This requires solution of the following eigenvalue problem,

$$
\mathbf{Q}_{m, \omega}^{*} \mathbf{W} \mathbf{Q}_{m, \omega} \boldsymbol{\Psi}_{m, \omega}=\boldsymbol{\Psi}_{m, \omega} \boldsymbol{\Lambda}_{m, \omega}
$$

where $\boldsymbol{\Lambda}_{m, \omega}$ is the diagonal eigenvalues matrix and $\boldsymbol{\Psi}_{m, \omega}$ is the matrix of orthogonal eigenvectors at the angular frequency $\omega$ and for the $m^{\text {th }}$ azimuthal mode. The term $\mathbf{W}$ represents the discretized weighting matrix, which will be defined in section III.B, and the superscript ${ }^{*}$ denotes the Hermitian transpose.

The $i^{t h}$ mode $\boldsymbol{\Phi}_{m, \omega}^{(i)}$ is computed by projecting the $i^{t h}$ eigenvector $\Psi_{m, \omega}^{(i)}$ on the data matrix and normalizing it by the corresponding eigenvalue $\lambda_{m, \omega}^{(i)}$ :

$$
\boldsymbol{\Phi}_{m, \omega}^{(i)}=\mathbf{Q}_{m, \omega} \boldsymbol{\Psi}_{m, \omega}^{(i)}\left(\lambda_{m, \omega}^{(i)}\right)^{-1 / 2}
$$

The mode $\boldsymbol{\Phi}_{m, \omega}^{(i)}$ represents the $i^{t h}$ most energetic mode at the angular frequency $\omega$ for the azimuthal mode $m$. As the data matrix is composed of the quantities $\left[u_{x}, u_{r}, u_{\theta}, p, s\right], \boldsymbol{\Phi}_{m, \omega}^{(i)}$ will contain the 2-D spatial modes of the velocity, pressure and entropy fields.

\section{B. Definition of the inner product}

In the SPOD formulation shown in equation 3 , the $\mathbf{Q}_{m, \omega}^{*} \mathbf{W} \mathbf{Q}_{m, \omega}$ term represents the inner product, which needs to be constructed by defining the matrix W. Rowley et al. [35] discussed the advantages of using an energy-based inner product which is a physical norm for analyzing a flow dataset with POD. The linear 'disturbance energy' [30] is an attractive candidate and can be expressed as a second order corollary:

$$
E_{d}=\underbrace{\frac{p_{f}^{2}}{2 \rho_{b} c_{b}^{2}}}_{E_{P}}+\underbrace{\frac{1}{2} \rho_{b} \mathbf{u}_{\mathbf{f}} \cdot \mathbf{u}_{\mathbf{f}}}_{E_{K}}+\underbrace{\frac{\rho_{b} T_{b} s_{f}^{2}}{2 c_{p, b}}}_{E_{S}}+\underbrace{\rho_{f} \mathbf{u}_{\mathbf{b}} \cdot \mathbf{u}_{\mathbf{f}}}_{E_{C}}+E_{Y},
$$

where the subscripts $b$ and ${ }_{f}$ denote the baseflow solution and the first order fluctuations, respectively. The first 3 terms respectively show the contributions from pressure, kinetic and entropy fluctuations while the term $E_{Y}$ is associated with mixture inhomogeneities and is algebraically very complex [30]. However, $E_{d}$ is not a norm created by any choice of inner product and so cannot be used directly in SPOD. On the other hand, it has been shown that the contributions from mixture inhomogeneities and the cross-term $E_{C}$ were small relative to other terms present in equation 5 [30, 36]. The following semi-norm is thus defined:

$$
\mathbf{Q}_{m, \omega}^{*} \mathbf{W} \mathbf{Q}_{m, \omega}=\frac{1}{2} \iiint_{\Omega} \mathbf{Q}_{m, \omega}^{*} \operatorname{diag}\left(\bar{\rho}, \bar{\rho}, \bar{\rho}, \frac{1}{\bar{\rho} \bar{c}^{2}}, \frac{\bar{\rho} \bar{T}}{\overline{c_{p}}}\right) \mathbf{Q}_{m, \omega} r d r d x d \theta
$$


where $\Omega$ represents the volume of interest.

\section{Definition of the acoustic sub-domain $\Omega$}

In the present study, the sub-domain $\Omega$ was chosen to be the region where the pressure term $E_{p}$ was larger than the other terms in the disturbance energy equation. To compare these terms, time averaged volume norms have been defined by computing the volume weighted time average of the modulus of the terms in equation 5 , so that,

$$
\left\|\hat{E}_{d, m}\right\|=\underbrace{\overline{\left|\hat{E}_{P, m}\right|} d V}_{\left\|\hat{E}_{P, m}\right\|}+\underbrace{\overline{\left|\hat{E}_{K, m}\right|} d V}_{\left\|\hat{E}_{K, m}\right\|}+\underbrace{\overline{\left|\hat{E}_{S, m}\right|} d V}_{\left\|\hat{E}_{S, m}\right\|}+\underbrace{\overline{\left|\hat{E}_{C, m}\right|} d V}_{\left\|\hat{E}_{C, m}\right\|} .
$$

The norm of the total disturbance energy at azimuthal mode $m$, noted $\left\|\hat{E}_{d, m}\right\|$, features a pressure term $\left\|\hat{E}_{P, m}\right\|$, a kinetic term $\left\|\hat{E}_{K, m}\right\|$, an entropy term $\left\|\hat{E}_{S, m}\right\|$ and a cross-term $\left\|\hat{E}_{C, m}\right\|$. The mixture inhomegeneity term was omitted for simplicity. The term $d V$ in equation 7 represents the local cell volume and $\left|\hat{E}_{P, m}\right|$, for instance, represents the modulus of the disturbance energy pressure term of mode $m$.

Figure 2 shows the radial profile of these norms at streamwise locations $x / D=5$ and $x / D=12$, for the $m=0$ azimuthal mode. While the entropy term is dominant in the flame region $(r / D<0.75)$, the kinetic energy term prevails in the near-field region, which widens downstream as the jet expands radially. The minimum radial value for which the pressure term is dominant therefore increases with streamwise location. Note that far from the jet, the pressure term in equation 5 represents the acoustic energy. Based on Fig. $2, \Omega$ was chosen to be the region $x / D<12$ and $r / D>6$ (shown in Fig. 11, where the acoustic energy is dominant.

It is worth noting that the semi-norm formulation shown in equation 6 uses a similar idea to the 'extended POD' [37, 38], which finds coherent structures contributing to the fluctuations of a given quantity in a particular sub-domain. Our formulation considers disturbance energy as the quantity of interest which, in the sub-domain $\Omega$, is primarily acoustic energy as demonstrated in Fig 2 Hence, our formulation shows the importance of different coherent structures in the sound generation process [39].

In order to assess which azimuthal modes are relevant for this study, the pressure norm term $\left\|\hat{E}_{P, m}\right\|$ in equation 7 is spatially integrated in the acoustic sub-domain $\Omega$ and is normalized by the axisymmetric mode value. This norm is displayed in Fig. 3 and shows that the acoustic energy decays rapidly as the azimuthal wavenumber increases. It decreases by more than an order of magnitude for $m=1$ and down to $10^{-4}$ for the fifth mode, meaning that approximately $90 \%$ of the acoustic energy is contained in the axisymmetric mode. Since this paper focuses on the contribution of coherent structures to combustion noise, the following results therefore analyze the axisymmetric mode only. 

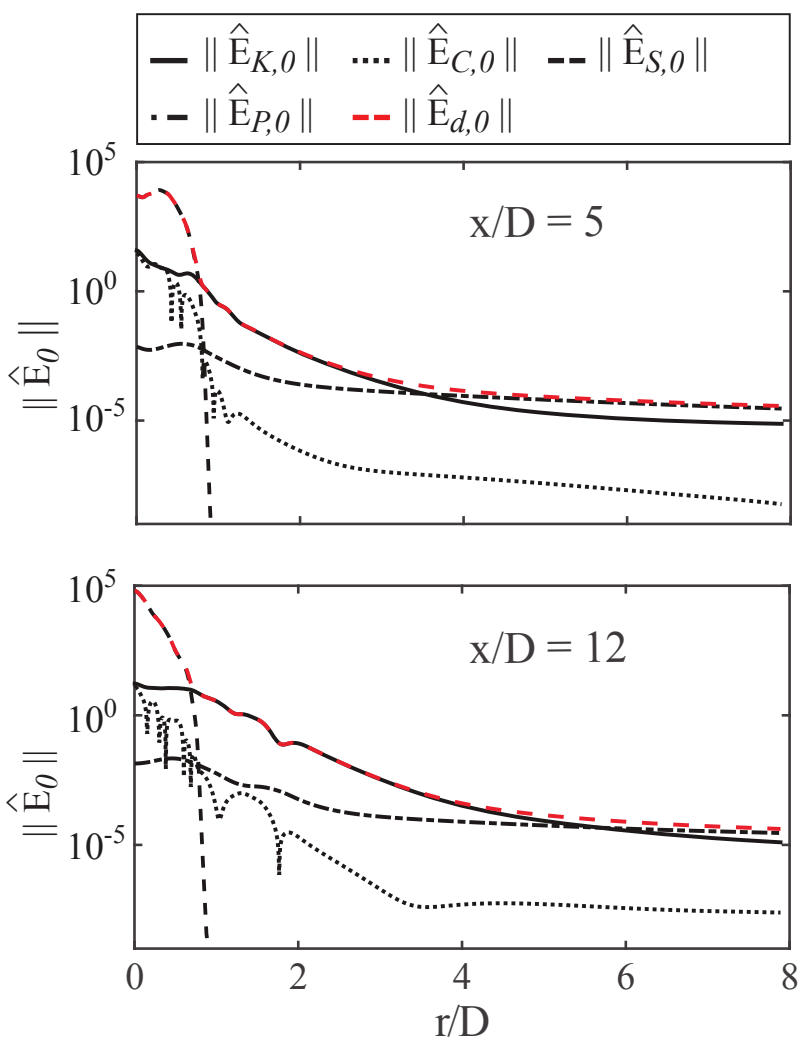

Fig. 2 Time averaged volume norm terms shown in equation 7 for the axisymmetric mode at two streamwise locations for the $\phi=1$ case.

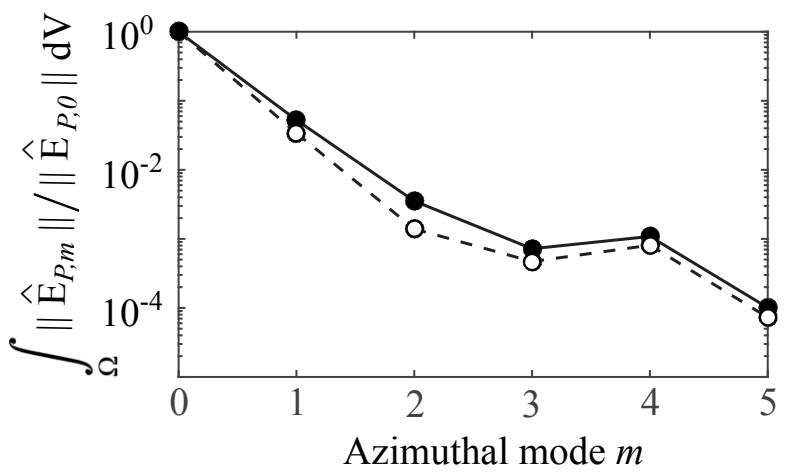

Fig. 3 Norm $\left\|\hat{E}_{P, m}\right\|$ integrated over the acoustic sub-domain $\Omega$ and normalized by the axisymmetric mode value for $\phi=1$ (full circles) and $\phi=0.7$ (hollow circles).

\section{Frequency-wavenumber diagrams and phase speed}

For a spatial mode $\boldsymbol{\Phi}_{m, \omega}^{(i)}$, a spatial Fourier transform along a given streamwise line will give the streamwise wavenumber content of the mode. In order to limit the gain loss, a 75\% cosine-tapered (Tukey) window [40] was used to perform this Fourier decomposition. Displaying the amplitude of the spatial Fourier transforms of the most energetic mode $\boldsymbol{\Phi}_{m, \omega}^{(1)}$ for all frequencies will effectively show the 'wavenumber-frequency' diagram of the dominant SPOD modes, which will be analyzed in section IV 


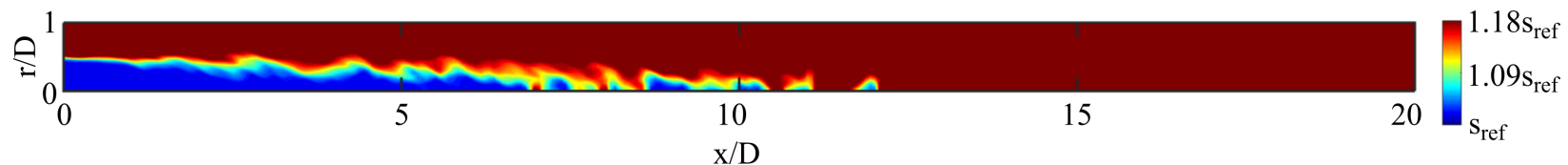

Fig. 4 Snapshot of axisymmetric component of entropy $\hat{s}_{0}(x, r, t)$. Animation can be found as supplemental material.

The wavenumber of maximum amplitude of mode $\boldsymbol{\Phi}_{m, \omega}^{(1)}$, noted $k_{\text {max }}$, will be used to get an estimation of the normalized phase velocity $v_{p} / \bar{u}_{r e f}$ of the mode structures. The phase velocity is computed using the following relation:

$$
v_{p} / \bar{u}_{r e f}=\omega D / k_{\max }
$$

\section{Results and discussion}

A snapshot of the axisymmetric component $(m=0)$ of the entropy field is first shown in Fig. 4 to provide some background on the dominant physical mechanisms and coherent structures expected to find in the SPOD modes. In the linear sense, $s^{\prime} / c_{p, b}=T^{\prime} / T_{b}-(\gamma-1) p^{\prime} / p_{b}[41]$ and therefore, entropy is a strong function of temperature across the flame. For this reason, the entropy field is chosen to analyze the flame structures.

Large deformations of the flame front are observed in the shear layer for $x / D<5$, induced by vortices arising from the Kelvin-Helmholtz (K-H) instability. Around the flame tip, smaller structures are identified around the centreline. They represent isolated pockets of unburnt mixture which are generated downstream of the unburnt core and are eventually consumed as they move further downstream. Coherent structures corresponding to both of these features will be identified by the SPOD modes and will be discussed later.

The SPOD results are now discussed in the following. As only the axisymmetric mode is analyzed, the subscript $m$ referring to the azimuthal mode number is omitted for readability. Figure 5 shows the eigenvalues $\lambda^{(i)}$ from equation 3 for the $\phi=1$ and $\phi=0.7$ cases. As the eigenvalues represent the amount of energy in several SPOD modes, this enables us to compare their relative importance. The difference between the $1^{\text {st }}$ and $2^{\text {nd }}$ mode (highlighted with the red shaded area) in the range $0.5<S t<1.2$ shows that the flame has a low rank behavior at those frequencies and peaks at $S t \approx 0.6$. At this frequency, the dominant mode contains more than $70 \%$ of the overall energy, for both $\phi$ cases. As discussed in section [1] the same low-rank behavior has been found in a subsonic non-reacting jet at higher Re [27]. The energy content of the dominant SPOD mode at different frequencies can also be extracted from the top spectrum in Fig. 5. As can be seen, most of the energy is contained at the lowest frequencies and decays strongly for $S t>1$. Even though the low frequencies are clearly the most energetic, it will still be interesting to analyze the SPOD results for $S t>1$ to have an overall view of the sound generation process.

Additional information on the SPOD modes $\boldsymbol{\Phi}_{m, \omega}^{(i)}$ can be obtained by analyzing their spatial shape. It is recalled 

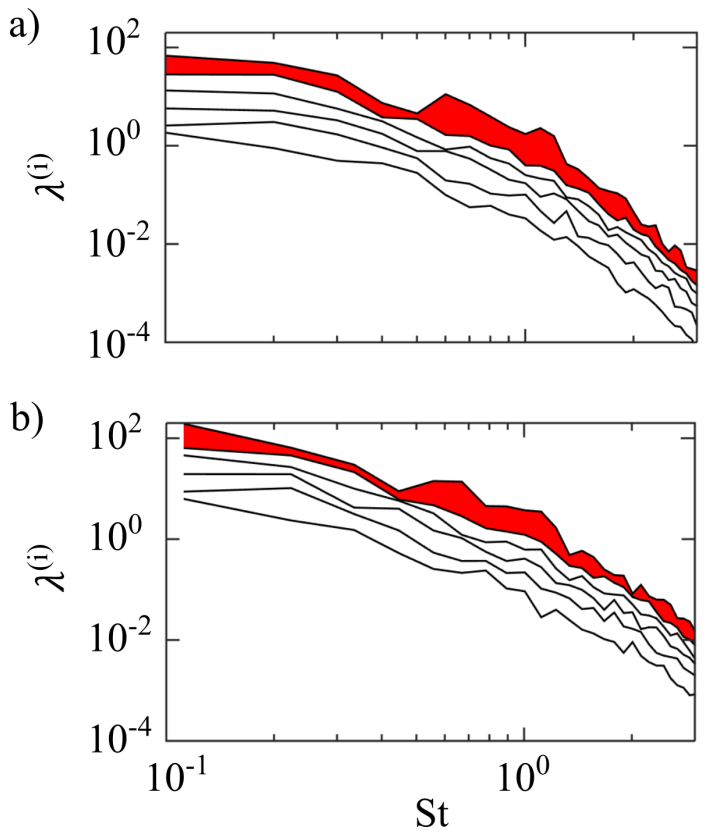

Fig. 5 SPOD eigenvalues $\lambda^{(i)}$ from equation 3 for a) $\phi=0.7$ and b) $\phi=1$. The red shaded area highlights the differences between the $1^{\text {st }}$ and $2^{\text {nd }}$ SPOD modes.

that the dominant SPOD modes identified using the inner product defined in section III.B are the ones that contribute the most to the acoustics. The entropy field of the most energetic SPOD modes is shown in Fig. 6 for various frequencies. An animated version of Fig. 6 6 provides a complete picture of how coherent structures evolve over time (see supplemental material). Both $\phi=1$ and $\phi=0.7$ flames were found to display very similar results and only the former case is shown for brevity. The dominant wavenumber of the coherent structures increases with frequency, as would be expected for a disturbance advecting with a nearly constant phase speed. At low frequencies, the dominant structures are those that emerge from the shear layer near the inlet and are convected downstream.

From the preliminary observations of the instantaneous entropy field in Fig. 4, it is concluded that these structures are arising from the K-H instability, which deforms the flame front in the shear layer. From the eigenvalues' analysis which showed the low-rank behavior of the flame, it can also be concluded that the mode shown for $S t=0.6$ in Fig. 6 and which features the K-H instability structures contains more than $70 \%$ of the energy at this frequency. However, these $\mathrm{K}-\mathrm{H}$ structures become less visible at higher frequencies where they seem to break down as they travel downstream. For instance at $S t \approx 0.9$, the initial shear layer perturbations are only identifiable until $x=3 D$.

At higher frequencies, the smaller structures are of similar lengthscale to the laminar flame thickness. Figure 6 shows that the coherent structures tend to be of larger amplitude around the centreline, downstream of the unburned core region. Considering the results shown in Fig. 4 indicates that these structures are associated with small flame dynamics and flame annihilations occurring in that region. These smaller features will therefore mostly contribute to the acoustics at higher frequencies. 


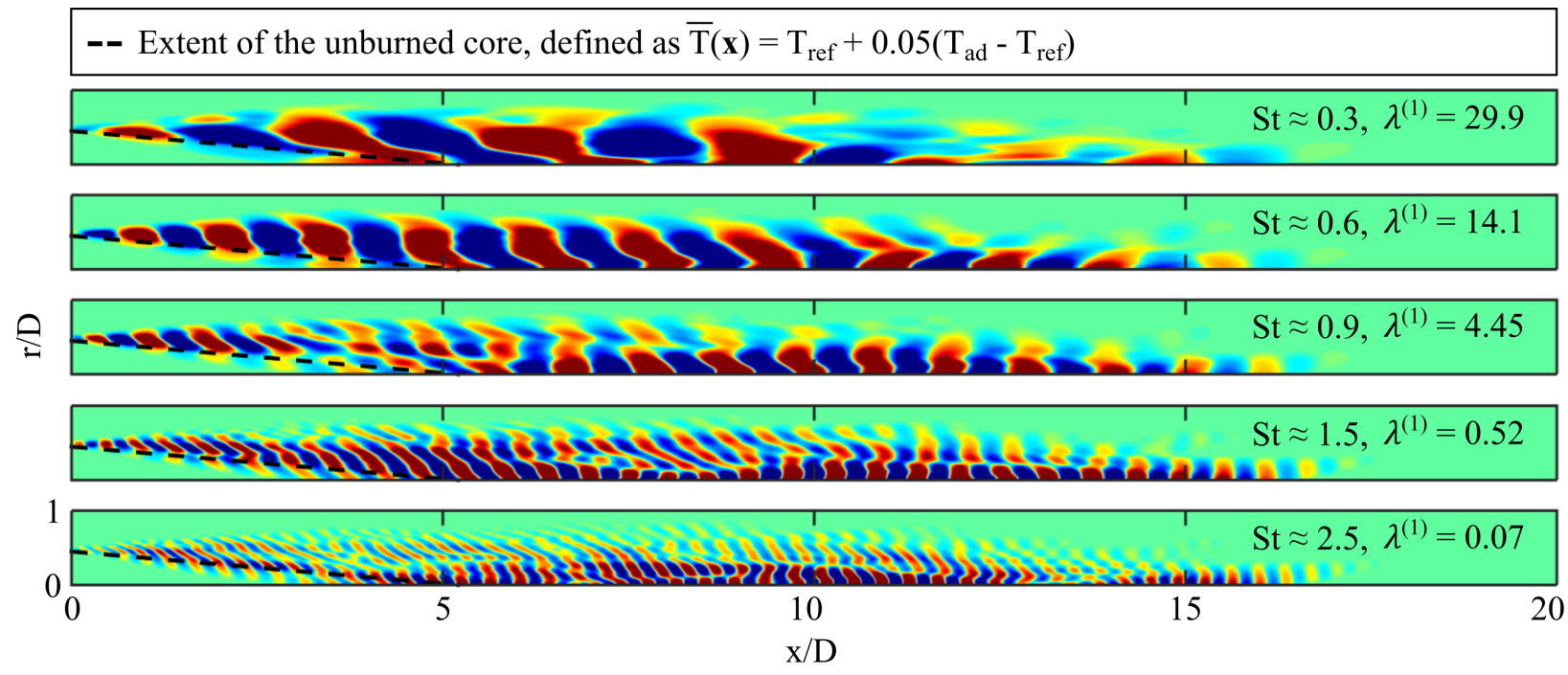

Fig. 6 Entropy field of the dominant SPOD mode for $\phi=1$ at several Strouhal numbers. Color scale shows $\pm 25 \%$ of the maximum value. Animations can be found as supplemental material.

These structures are also interesting to analyze from a different perspective. Tissot et al. [29] and Schmidt et al. [27] noted the presence of similar coherent structures downstream of the potential core of non-reacting jets, in the streamwise velocity field and in the pressure field, respectively. Tissot et al. [28] suggested that they were the result of non-modal growth, seeded by non-linearities through the Orr mechanism. This interpretation is consistent with the highly non-linear behavior of small flame dynamics occurring downstream of the unburned core of the jet. Overall, these results suggest a multi-modal behavior with dominant structures in the shear layer at lower frequencies, that are arising from the K-H instability. The other features identified along the jet centreline are representative of the small non-linear flame dynamics. They are evident in a broad range of frequencies but most visible at higher frequencies.

Further characteristics of the structures can be found by analyzing their propagation speed. Figure 7 shows the structures' phase velocity $v_{p} / \bar{u}_{r e f}$, defined in equation 8 , for both equivalence ratios along the centreline $[0<x / D<20 ; r / D=0]$ and lip-line $[0<x / D<5 ; r / D=0.5]$. The centreline structures travel at speeds around $90 \%$ of the inlet velocity, which is close to the average streamwise velocity inside the flame, as shown in Fig. 8 . On the other hand, the estimated velocity of the shear layer structures is around $0.75 \bar{u}_{r e f}$. This latter phase speed is similar to that found by Moore [42] and Schmidt et al. [27] for cold jet instabilities at subsonic Mach numbers, confirming that the same shear layer mechanism is present in the flames studied here.

The pressure field of the most energetic SPOD modes can provide complementary information on the hydrodynamic and acoustic behavior of the jet, as demonstrated in Fig. 9 and in the supplemental material. As discussed in section [1] wavepacket structures in the shear layer have been linked to the K-H instability in non-reacting jets [24]. These structures can be seen at $S t \approx 0.3$ for $\phi=0.7$, confirming that the K-H instability is present in reacting jets as well. The $\phi=1$ wavepackets are less organized, potentially indicating that the K-H instability is not as important under 


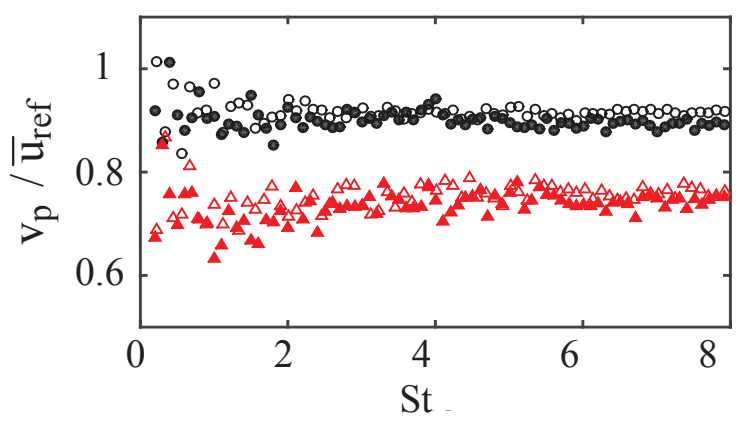

Fig. 7 Phase velocity of entropy's coherent structures at the jet centreline (black circles) and in the shear layer at $\mathbf{r} / \mathrm{D}=\mathbf{0 . 5}$ (red triangles) for $\phi=0.7$ (full markers) and $\phi=1$ (hollow markers).

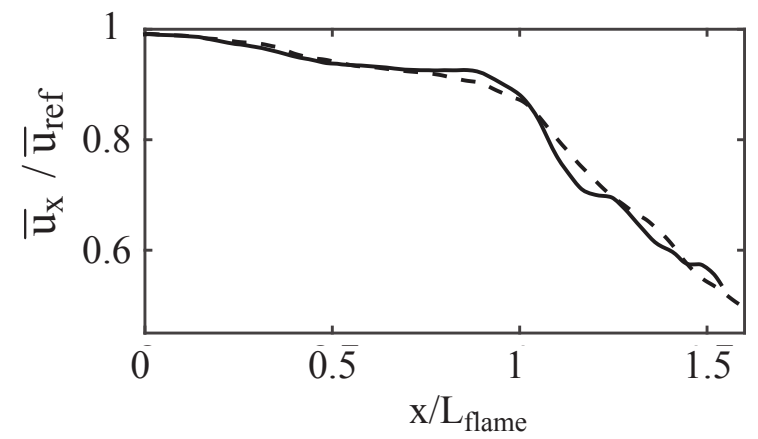

Fig. 8 Mean streamwise velocity at jet centreline for $\phi=1$ (solid line) and $\phi=0.7$ (dashed line).

stoichiometric conditions. Indeed, several studies have shown that shear layer instabilities become less pronounced as the temperature ratio increased [43, 44].

The animated version of Fig. 9 in the supplementary material shows the acoustic waves generated by these flames more clearly. The near-nozzle region seems to be a strong source of noise for $S t \approx 0.3$ and 0.6 in both flames. However, at $S t \approx 3$, the noise sources are spread out all over the flame brush, with a dominant beam emerging from around $x / D=5$ and several other smaller sources. As expected, the $\mathrm{K}-\mathrm{H}$ structures emerging from the inlet are therefore responsible for the sound generated by the flame at low frequencies. The centreline structures, which have been connected to small flame dynamics, are spatially more spread and are dominant at higher frequencies.

In order to provide an overall view of the structures present in the jet for a wide range of frequencies, Fig. 10 shows pressure wavenumber-frequency diagrams of the most energetic SPOD mode. For a given frequency, these diagrams represent the amplitude of the spatial Fourier transform performed at a streamwise segment of the pressure field of the SPOD modes. These diagrams therefore provide information on the dominant wavenumbers of the structures at different frequencies. The segment $0<x<0.8 L_{\text {flame }}$ along the jet centreline has been used for the top row in Fig. 10 while the bottom row represents the results at the lip-line. Three distinct features can be observed. First, acoustic waves travelling at the speed of sound can be seen for both cases but are weaker in amplitude for $\phi=0.7$. Second, structures travelling at $90 \%$ of the inlet velocity are also observed at the centreline. The phase velocity shown in Fig. 7 suggests 
$\phi=0.7$
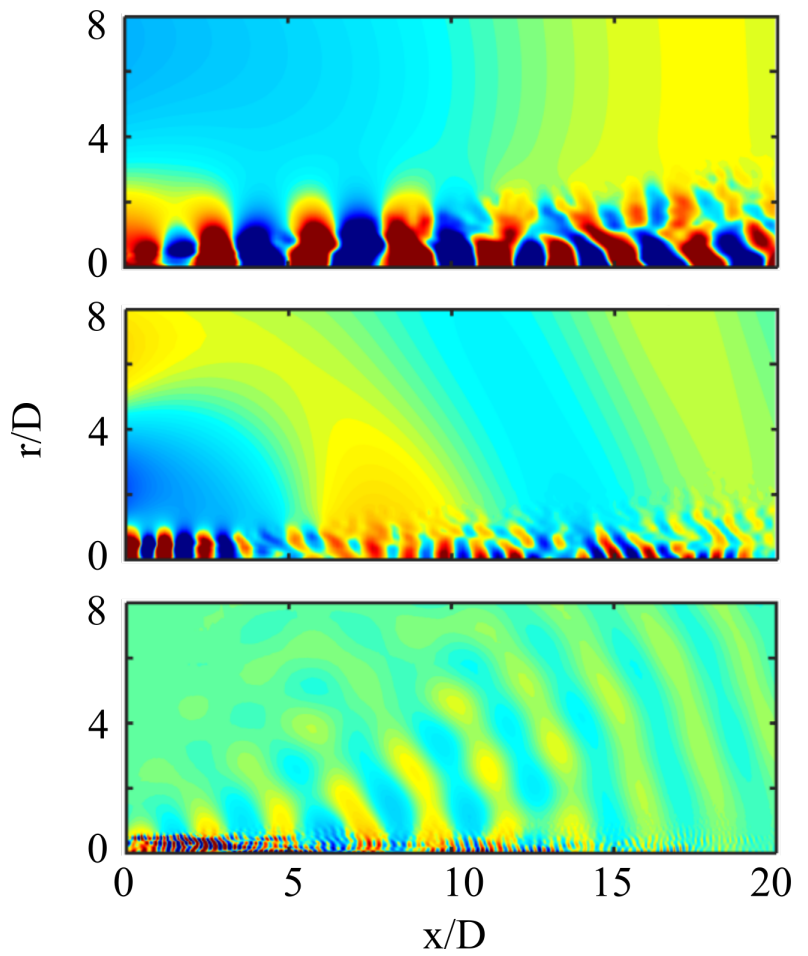

$\phi=1$
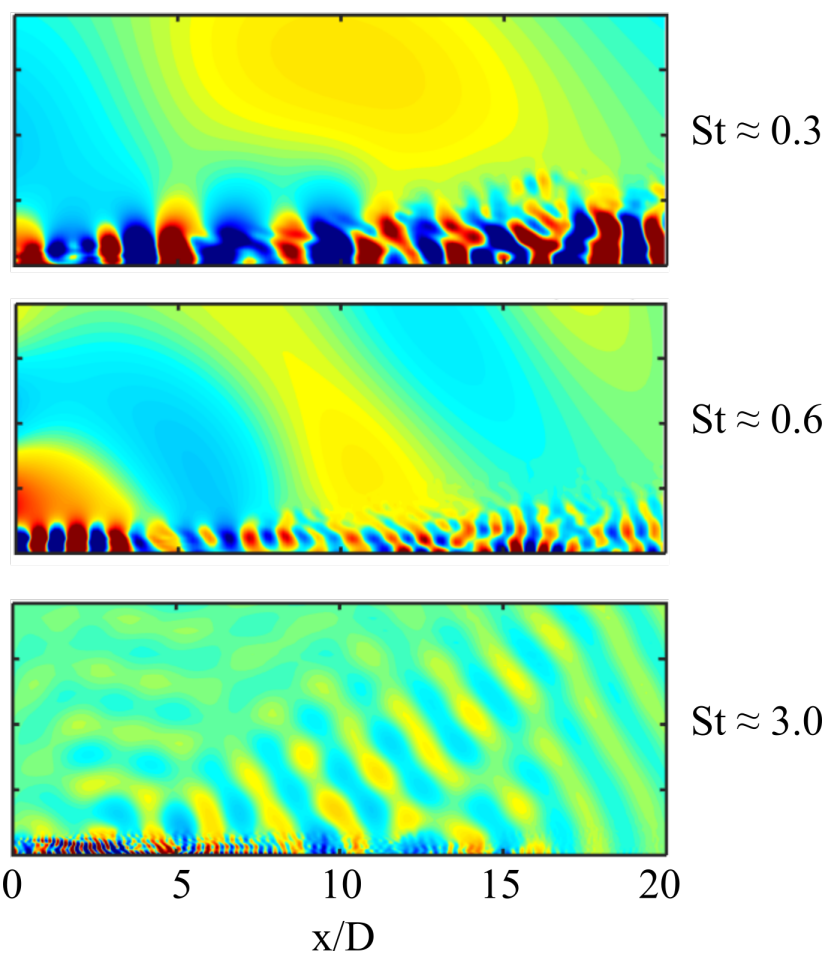

Fig. 9 Pressure field of the dominant SPOD mode for $\phi=0.7$ and $\phi=1$, at several Strouhal numbers. Color scale shows $\pm 25 \%$ of maximum value. Animations can be found as supplemental material.

that they are representative of the centreline structures previously identified in the entropy field and are therefore related to small flame dynamics and to the Orr mechanism. These features have a very strong signature for $S t>2$, even though we previously observed them at frequencies $S t \approx 0.9$ in Fig. 6 The wavenumber-frequency diagram at $r / D=0.5$ highlights the signature of shear layer structures travelling at a speed close to $0.75 \bar{u}_{r e f}$. These structures are dominant at $S t<1$, as shown by the high amplitude on the bottom left corner of the diagrams. Overall, these diagrams show that the shear layer structures near the inlet can have a significant contribution to the far-field noise for $S t<1$ (Fig. 10 b), while non-linear small flame dynamics are more important at $S t>2$ (Fig. 10 a).

\section{Conclusions}

DNS data of two turbulent premixed flames featuring sound generation were analyzed using a spectral proper orthogonal decomposition (SPOD) method. By defining a specific inner product, we were able to identify the flame and jet coherent structures that correlated with the flame acoustics. The SPOD inner product took into account the kinetic, entropy and acoustic fluctuations through a linearized disturbance energy formulation. An azimuthal decomposition showed that the pressure fluctuations in the far-field were dominated by the axisymmetric mode, justifying analysis of only this mode in this study. 

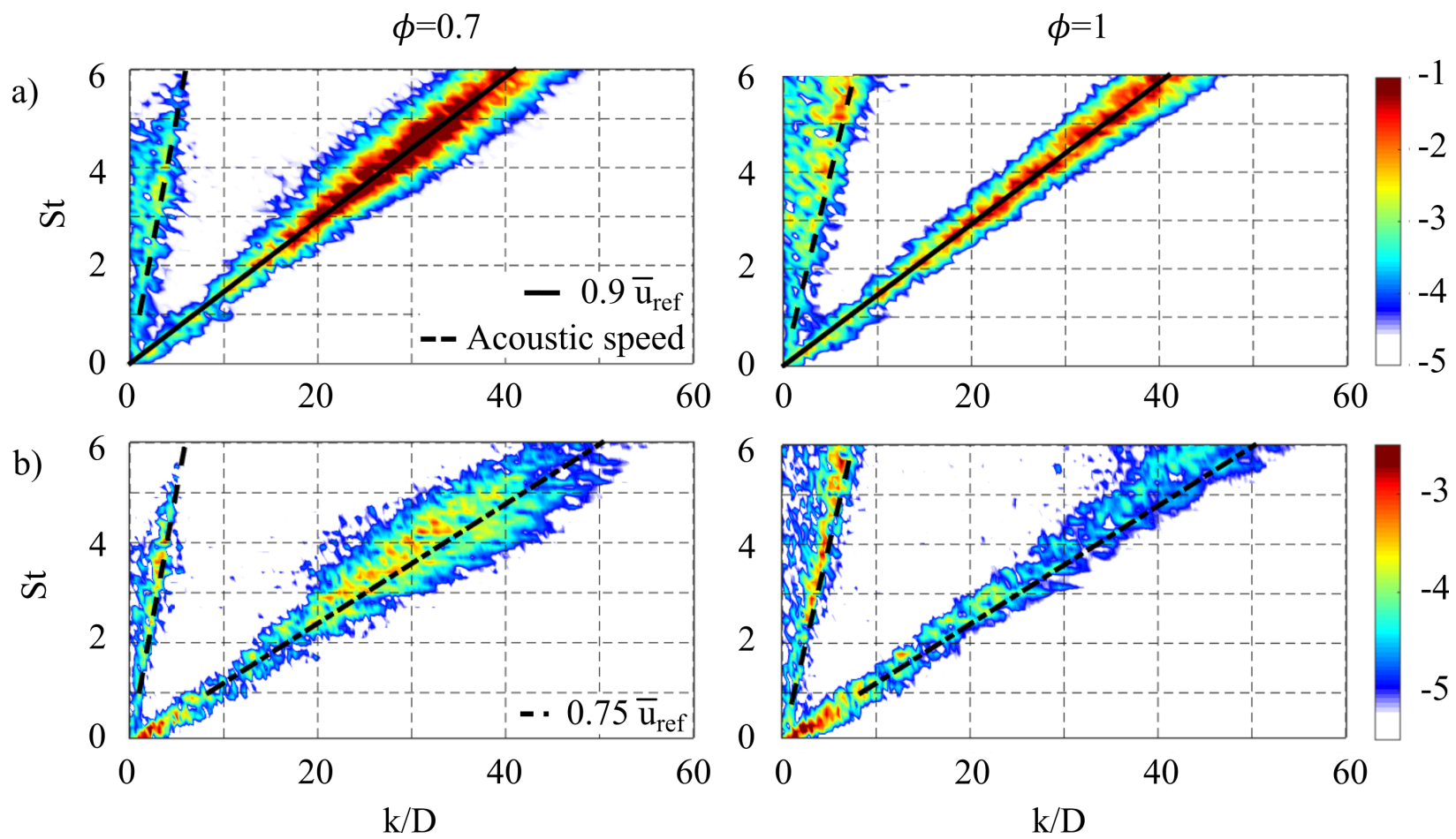

Fig. 10 Pressure wavenumber-frequency diagrams of the dominant SPOD mode for $\phi=0.7$ and $\phi=1$ at a) centreline and b) lip-line. The color axis is a logarithmic scale.

Two main types of axisymmetric coherent structures contributing to the far-field sound were then found. The first type of structures was originating from the shear layer and was linked to the $\mathrm{K}-\mathrm{H}$ instability. These structures produced sound through deformation of the flame front in the shear layer. They contained most of the overall acoustic energy and were dominant at Strouhal numbers $S t<1$.

The second type of structures was linked to small non-linear flame dynamics and to the Orr mechanism. These structures were concentrated near the jet centreline and downstream of the potential core where large fluctuations of flame surface area were present. They traveled at a speed close to the inlet mean velocity and were more important at $S t>2$. Regardless of their energy content, both types of structures have important contributions to the broadband nature of combustion noise.

The present study showed that SPOD can be applied to identify structures responsible for sound generation in reacting flows. This approach can also be used to study diffusion flames or premixed flames with different Karlovitz/Damkohler numbers, leading to an improved understanding of physical mechanisms responsible for combustion noise.

\section{Acknowledgements}

This work was supported by the Australian Research Council (ARC) [grants DP120101830 and DE180100416] and the University of Melbourne through a Melbourne International Research Scholarship and a Melbourne International Fee Remission Scholarship. The research benefited from computational resources provided through the National 
Computational Merit Allocation Scheme, supported by the Australian Government. The computational facilities supporting this project included the Australian NCI National Facility and the Pawsey Supercomputing Center.

\section{References}

[1] Nicoud, F., and Poinsot, T., "Thermoacoustic instabilities: Should the Rayleigh criterion be extended to include entropy changes?" Combustion and Flame, Vol. 142, No. 1-2, 2005, pp. 153-159. doi:https://doi.org/10.1016/j.combustflame.2005.02.013.

[2] Lieuwen, T., "Modeling Premixed Combustion-Acoustic Wave Interactions: A Review," Journal of Propulsion and Power, Vol. 19, No. 5, 2003, pp. 765-781. doi:https://doi.org/10.2514/2.6193.

[3] Briffa, F., Clark, C. J., and Williams, G. T., “Combustion noise," Journal of the Institute of Fuel, Vol. 46, No. 386, 1973, pp. $207-216$.

[4] Hassan, H. A., "Scaling of combustion-generated noise," Journal of Fluid Mechanics, Vol. 66, No. 3, 1974 , pp. $445-453$. doi:https://doi.org/10.1017/S0022112074000292.

[5] Kotake, S., and Takamoto, K., "Combustion noise: Effects of the shape and size of burner nozzle," Journal of Sound and Vibration, Vol. 112, 1987, pp. 345-354. doi:https://doi.org/10.1016/S0022-460X(87)80201-8.

[6] Rajaram, R., and Lieuwen, T., “Acoustic radiation from turbulent premixed flames,” Journal of Fluid Mechanics, Vol. 637, 2009, pp. 357-385. doi:https://doi.org/10.1017/S0022112009990681.

[7] Tam, C. K. W., “The spectral shape of combustion noise,” International Journal of Aeroacoustics, Vol. 14, No. 3, 2015 , pp. 431-456. doi:https://doi.org/10.1260/1475-472X.14.3-4.431.

[8] Strahle, W. C., "A more modern theory on combustion noise," Recent Advances in the Aerospace Sciences, Springer, 1985, pp. $103-114$.

[9] Candel, S., "Combustion dynamics and control: progress and challenges," Proceedings of the Combustion Institute, Vol. 29, 2002, pp. 1-28. doi:https://doi.org/10.1016/S1540-7489(02)80007-4.

[10] Candel, S., Durox, D., and Schuller, T., "Flame interactions as a source of noise and combustion instabilities," 10th AIAA/CEAS Aeroacoustics Conference, 2004, p. 2928. doi:https://doi.org/10.2514/6.2004-2928.

[11] Schlimpert, S., Koh, S. R., Pausch, K., Meinke, M., and Schröder, W., “Analysis of combustion noise of a turbulent premixed slot jet flame," Combustion and Flame, Vol. 175, 2016, pp. 292-306. doi:https://doi.org/10.1016/j.combustflame.2016.

[12] Wiseman, S. M., Brear, M. J., Gordon, R. L., and Marusic, I., "Measurements from flame chemiluminescence tomography of forced laminar premixed propane flames," Combustion and Flame, Vol. 183, 2017, pp. 1-14. doi:https://doi.org/10.1016/j. combustflame.2017.05.003.

[13] Talei, M., Brear, M. J., and Hawkes, E. R., "Sound generation by laminar premixed flame annihilation," Journal of Fluid Mechanics, Vol. 679, 2011, pp. 194-218. doi:https://doi.org/10.1017/jfm.2011.131. 
[14] Talei, M., Hawkes, E. R., and Brear, M. J., "A direct numerical simulation study of frequency and Lewis number effects on sound generation by two-dimensional forced laminar premixed flames," Proceedings of the Combustion Institute, Vol. 34, No. 1, 2013, pp. 1093-1100. doi:https://doi.org/10.1016/j.proci.2012.07.034.

[15] Haghiri, A., Talei, M., Brear, M. J., and Hawkes, E. R., "Sound generation by turbulent premixed flames," Journal of Fluid Mechanics, Vol. 843, 2018, pp. 29-52. doi:https://doi.org/10.1017/jfm.2018.115.

[16] Brouzet, D., Haghiri, A., Talei, M., and Brear, M. J., “Annihilation events topology and their generated sound in turbulent premixed flames," Combustion and Flame, Vol. 204, 2019, pp. 268-277. doi:https://doi.org/10.1016/j.combustflame.2019.02. 028.

[17] Taira, K., Brunton, S. L., Dawson, S., Rowley, C. W., Colonius, T., McKeon, B. J., Schmidt, O. T., Gordeyev, S., Theofilis, V., and Ukeiley, L. S., "Modal analysis of fluid flows: An overview," AIAA Journal, 2017, pp. 4013-4041. doi:https://doi.org/10.2514/1.J056060.

[18] Kabiraj, L., Saurabh, A., Nawroth, H., and Paschereit, C. O., "Recurrence analysis of combustion noise," AIAA Journal, Vol. 53, No. 5, 2015, pp. 1199-1210. doi:https://doi.org/10.2514/6.2014-0623.

[19] Ghani, A., Poinsot, T., Gicquel, L., and Staffelbach, G., "LES of longitudinal and transverse self-excited combustion instabilities in a bluff-body stabilized turbulent premixed flame," Combustion and Flame, Vol. 162, No. 11, 2015, pp. 4075-4083. doi:https://doi.org/10.1016/j.combustflame.2015.08.024.

[20] Aoki, K., Shimura, M., Ogawa, S., Tanahashi, M., and Miyauchi, T., "Short and long-term dynamic modes of turbulent swirling premixed flame in a cuboid combustor," Proceedings of the Combustion Institute, Vol. 35, No. 3, 2015 , pp. $3209-3217$. doi:https://doi.org/10.1016/j.proci.2014.10.003.

[21] Towne, A., Schmidt, O. T., and Colonius, T., "Spectral proper orthogonal decomposition and its relationship to dynamic mode decomposition and resolvent analysis," Journal of Fluid Mechanics, Vol. 847, 2018, pp. 821-867. doi:https://doi.org/10.1017/ jfm.2018.283.

[22] Citriniti, J., and George, W. K., "Reconstruction of the global velocity field in the axisymmetric mixing layer utilizing the proper orthogonal decomposition,” Journal of Fluid Mechanics, Vol. 418, 2000, pp. 137-166. doi:https://doi.org/10.1017/ S0022112000001087.

[23] Gudmundsson, K., and Colonius, T., "Instability wave models for the near-field fluctuations of turbulent jets," Journal of Fluid Mechanics, Vol. 689, 2011, pp. 97-128. doi:https://doi.org/10.1017/jfm.2011.401.

[24] Jordan, P., and Colonius, T., "Wave packets and turbulent jet noise,” Annual Review of Fluid Mechanics, Vol. 45, 2013 , pp. 173-195. doi:https://doi.org/10.1146/annurev-fluid-011212-140756.

[25] Rodríguez, D., Sinha, A., Brès, G. A., and Colonius, T., "Inlet conditions for wave packet models in turbulent jets based on eigenmode decomposition of large eddy simulation data," Physics of Fluids, Vol. 25, No. 10, 2013, p. 105107. doi: https://doi.org/10.1063/1.4824479. 
[26] Schmidt, O. T., Towne, A., Colonius, T., Cavalieri, A., Jordan, P., and Bres, G. A., "Wavepackets and trapped acoustic modes in a Mach 0.9 turbulent jet: a global stability analysis," Journal of Fluid Mechanics, Vol. 825, 2017, pp. 1153-1181. doi:https://doi.org/10.1017/jfm.2017.407.

[27] Schmidt, O. T., Towne, A., Rigas, G., Colonius, T., and Brès, G. A., "Spectral analysis of jet turbulence," Journal of Fluid Mechanics, Vol. 855, 2018, pp. 953-982. doi:https://doi.org/10.1017/jfm.2018.675.

[28] Tissot, G., Lajús, F., Cavalieri, A., and Jordan, P., "Wave packets and Orr mechanism in turbulent jets," Physical Review Fluids, Vol. 2, No. 9, 2017, p. 093901. doi:https://doi.org/10.1103/PhysRevFluids.2.093901.

[29] Tissot, G., Zhang, M., Lajús, F., Cavalieri, A., and Jordan, P., "Sensitivity of wavepackets in jets to nonlinear effects: The role of the critical layer," Journal of Fluid Mechanics, Vol. 811, 2017, pp. 95-137. doi:https://doi.org/10.1017/jfm.2016.735.

[30] Brear, M. J., Nicoud, F., Talei, M., Giauque, A., and Hawkes, E. R., "Disturbance energy transport and sound production in gaseous combustion,” Journal of Fluid Mechanics, Vol. 707, 2012, pp. 53-73. doi:https://doi.org/10.1017/jfm.2012.264.

[31] Chen, J. H., Choudhary, A., De Supinski, B., DeVries, R., Hawkes, E. R., Klasky, S., Liao, W. K., Ma, K. L., Mellor-Crummey, J., Podhorszki, N., Sankaran, R., Shende, S., and Yoo, C. S., "Terascale direct numerical simulations of turbulent combustion using S3D," Computational Science and Discovery, Vol. 2, No. 1, 2009, p. 015001. doi:https://doi.org/10.1088/1749-4699/2/1/015001.

[32] Karami, S., Hawkes, E. R., Talei, M., and Chen, J. H., "Mechanisms of flame stabilisation at low lifted height in a turbulent lifted slot-jet flame,” Journal of Fluid Mechanics, Vol. 777, 2015, pp. 633-689. doi:https://doi.org/10.1017/jfm.2015.334.

[33] Peters, N., “The turbulent burning velocity for large-scale and small-scale turbulence,” Journal of Fluid Mechanics, Vol. 384, 1999, pp. 107-132. doi:https://doi.org/10.1017/S0022112098004212.

[34] Welch, P., "The use of fast Fourier transform for the estimation of power spectra: a method based on time averaging over short, modified periodograms," IEEE Transactions on Audio and Electroacoustics, Vol. 15, No. 2, 1967, pp. 70-73. doi:https://doi.org/10.1109/TAU.1967.1161901.

[35] Rowley, C. W., Colonius, T., and Murray, R. M., "Model reduction for compressible flows using POD and Galerkin projection," Physica D: Nonlinear Phenomena, Vol. 189, No. 1-2, 2004, pp. 115-129. doi:https://doi.org/10.1016/j.physd.2003.03.001.

[36] Giauque, A., Poinsot, T., Brear, M. J., and Nicoud, F., "Budget of disturbance energy in gaseous reacting flows," Proceedings of the Summer Program, Center for Turbulence Research, 2006, pp. 285-297.

[37] Maurel, S., Borée, J., and Lumley, J. L., "Extended proper orthogonal decomposition: Application to jet/vortex interaction," Flow, Turbulence and Combustion, Vol. 67, No. 2, 2002, pp. 125-136. doi:https://doi.org/10.1023/A:1014050204350.

[38] Borée, J., "Extended proper orthogonal decomposition: A tool to analyse correlated events in turbulent flows," Experiments in Fluids, Vol. 35, No. 2, 2003, pp. 188-192. doi:https://doi.org/10.1007/s00348-003-0656-3.

[39] Freund, J. B., and Colonius, T., “Turbulence and sound-field POD analysis of a turbulent jet," International Journal of Aeroacoustics, Vol. 8, No. 4, 2009, pp. 337-354. doi:https://doi.org/10.1260/147547209787548903. 
[40] Harris, F. J., "On the use of windows for harmonic analysis with the discrete Fourier transform," Proceedings of the IEEE, Vol. 66, 1978, pp. 51-83. doi:https://doi.org/10.1109/PROC.1978.10837.

[41] Chu, B., "On the energy transfer to small disturbances in fluid flow (Part I)," Acta Mechanica, Vol. 1, No. 3, 1965 , pp. $215-234$. doi:https://doi.org/10.1007/BF01387235.

[42] Moore, C. J., "The role of shear-layer instability waves in jet exhaust noise,” Journal of Fluid Mechanics, Vol. 80, 1977, pp. 321-367. doi:https://doi.org/10.1017/S0022112077001700.

[43] Trouve, A., Candel, S., and Daily, J. W., "Linear stability of the inlet jet in a ramjet combustor," 26th Aerospace Sciences Meeting, 1988, p. 149. doi:https://doi.org/10.2514/6.1988-149.

[44] Schlimpert, S., Koh, S. R., Feldhusen, A., Roidl, B., Meinke, M., and Schröder, W., "Hydrodynamic instability and shear layer effect on the sound emission of a turbulent jet flame," 21st AIAA/CEAS Aeroacoustics Conference, 2015, p. 2380. doi:https://doi.org/10.2514/6.2015-2380. 